\title{
Delegating Management Tasks through Negotiation and Contracting in the Open Service Market
}

\author{
Fernando Luís Dotti*
}

Instituto de Informática

Pontificia Universidade Católica do Rio Grande do Sul

Av. Ipiranga 6681, CEP 90619-900, Porto Alegre - RS, Brazil

telephone /fax: +55513203611/+55513203621

e-mail: fldotti@inf.pucrs.br

\begin{abstract}
With the aim of reducing the complexity and overhead of management activities to Customers of information systems, this work proposes the delegation of management tasks between autonomous administrative domains. This delegation is modelled considering the emerging electronic service market, such that Customers may selectively delegate management tasks over their resources to various Management Service Providers.

In order to establish the co-operation for management delegation while maintaining the autonomy of Customers and Providers, an approach based on negotiation is followed. The result of this negotiation is a contract which is enforced during service provisioning. This paper concentrates on the proposed contract and its negotiation. Due to the straight relation of the mechanisms here developed with electronic commerce efforts, the generalisation of the results here described should contribute to put forward electronic commerce.
\end{abstract}

Keywords

negotiation, contract, delegation, management tasks, open service market, inter-domain, autonomy.

\footnotetext{
* The author was partially sponsored by CNPq-Brazil (Grant number: 200752/92-8).
} 


\section{INTRODUCTION}

The management of Information Systems is a complex activity. Some reasons for this complexity are: the rich diversity of management aspects and of resources to be managed, and the consequently specialised knowledge needed to perform management; the constant introduction of new technologies in organisations due to the fast obsolescence of information technologies; and the increasing flexibility of modern organisations, whose strategies require high adaptability from the information systems and their management.

Considering this, the necessity for forms of operationalizing management activities arises to alleviate the complexity and overhead for the organisation using the information system, and to allow more flexibility in selecting and introducing new technologies. This work proposes the delegation of management tasks between distinct autonomous administrative domains as a new form to address these issues. This delegation consists in the establishment and administration of a form of cooperation where one administrative domain assumes management tasks on resources from another administrative domain. This should: help customers to reduce in-house overhead and management knowledge; support the fast introduction of new solutions; and assist the establishment of inter-domain cooperations. With this, technical support for organisational strategies is provided.

However, in order to achieve that, automated support for ease creation and manipulation of management delegation co-operations must be provided. With this, not only customers will more often choose to delegate management activities in its premises, but also the emergence of service providers is fostered, resulting in a diversity of management services tailored for the most distinct customer needs, and in competition in the open service market.

This paper concentrates on the automatic support proposed and implemented for establishing management delegation co-operations. This involves: the definition of a business model relating customer and provider domains; the identification and modelling of the information exchanged by and processed in the involved domains; the identification and modelling of inter-domain co-operating components; and the consideration of interactions with auxiliary roles in the service market. Requirements for the underlying platform in order to implement the proposed mechanisms are discussed as well.

\section{BUSINESS MODEL}

An electronic service market is emerging out of the efforts of individuals, enterprises, international fora, and other organisations working for the establishment of a common infrastructure for electronic information exchange. Each of these entities participates in this environment by attaching its own information system to a global communication infrastructure and by interoperating with some basic mechanisms adopted in the environment. In the context of this work, these entities will be represented by administrative domains, because they 
own information systems and have the autonomy to decide upon their configuration and utilisation.

An administrative domain in the electronic environment can reach and is reachable by another domains also in the environment. Out of the interaction among domains, relationships may be established, where domains play defined roles. Role definitions currently available are: Network Provider, Service Provider, and Customers.

The delegation of management tasks is modelled considering the emerging electronic service market, and is represented through a customer-provider relationship. In this relationship the provider assumes the management tasks which the customer delegates on its own resources. Due to their autonomy, the distinct administrative domains are considered to be heterogeneous in various aspects, for instance: resources used, security and authority concession policies, management services and features offered by the provider and needed by customers, etc. In order to achieve an agreement between the involved domains and at the same time consider these issues, an approach based on negotiation is followed. The negotiation establishes the various terms of a contract ruling the management delegation co-operation and, after the contract is achieved, both customer and provider will be responsible to enforce the contract terms.

In Figure 1 the inner ellipsis represents the target co-operation to be achieved: the Customer owns resources to be managed and wants to use high-level management functionality on these resources without being involved with the operation of the management services. The Service Provider provides the management service on the resources of the customer and offers the desired functionality to the Customer. In order to achieve that, i.e. in order to establish the co-operation depicted in the inner ellipsis, a co-operation of other roles is necessary. This second co-operation is depicted by the outer ellipsis in Figure 1, where general roles from the open service market are included (Notary, Payment, etc.) and two roles are proposed at Customer and Provider domains: the Customer and Provider Contract Managers. The Contract Managers are responsible in their respective domains for the negotiation and enforcing of contracts representing the management delegation cooperation. These roles are further detailed in this paper.

Some issues identified in the business model lead to high-level requirements to be fulfilled by the Contract Managers:

- support the establishment of co-operations among previously unknown parties. In such cases providers have no a priori knowledge and authorisation on customer resources;

- allow selective delegation, i.e. it must be possible to delegate specific management activities over specific groups of resources of the customer;

- treat multi-provider and multi-manager situations. In such situations, conflicts between mangers may take place. Conflicts may have to be handled during cooperation establishment and/or operation; 
- support uniform procedures to perform delegation of computing and communication management activities;

- support reversibility of delegation (substitution of provider). The customer which is not satisfied with the services of a provider has the possibility of negotiating the provision of the management task with another provider in the same area, and cancelling the agreements with the first one.

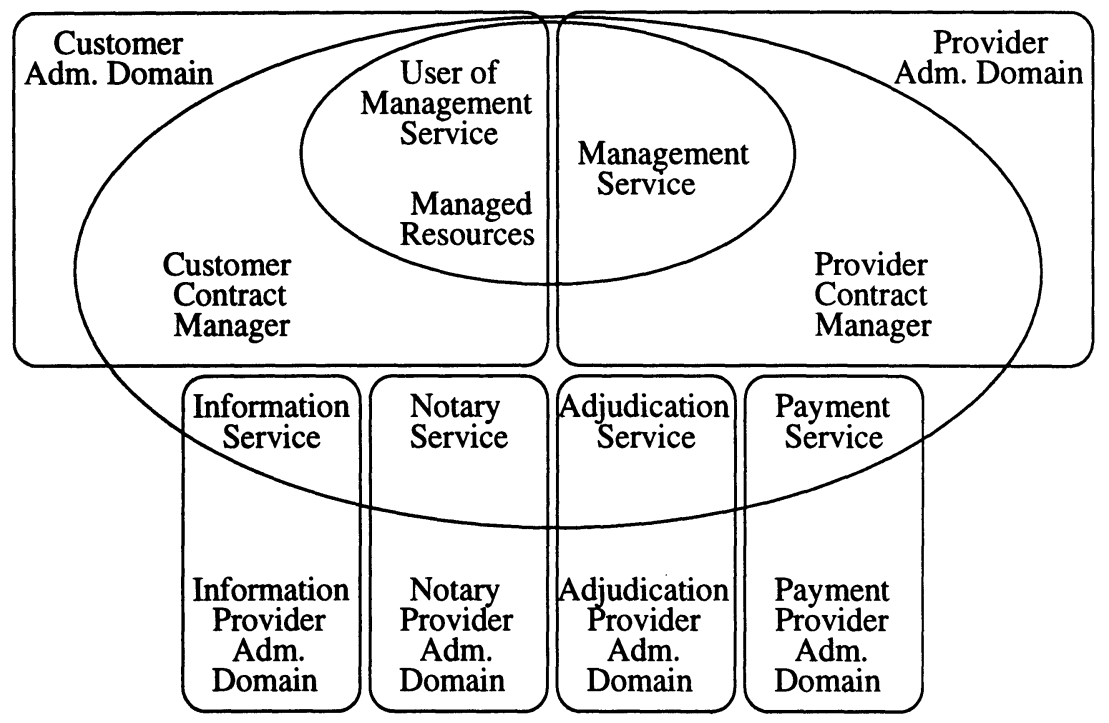

Figure 1: Business Model

\section{CO-OPERATION ESTABLISHMENT}

In this section a brief description of the activities of each phase of the co-operation is given. After that, kinds of information exchanged among the domains, as well as communicating components are described.

\subsection{Description in phases}

Contract Managers are independent entities which may contact each other in the service market, establish co-operations, last an arbitrary period of time in this cooperation, and finish it. In this section, a time ordered description of the main activities in the life-cycle of a co-operation is given. This description is divided in three main phases related to the provision of the management service: pre-service, in-service, and post-service.

\section{Pre-service Phase}

Service Advertisement: Providers advertise their management services in the service market through the Trader, Directory, WWW, etc.

Identification of management tasks to be delegated: Responsible humans on the customer side, while identifying management activities which have to be 
performed at the customers information infrastructure, should take into consideration the services offered in the market and delegate some of the identified management activities. Providers may also propose management delegation as a part of another service offer (for instance, VPN providers which assume also some management tasks at the Customer Premises Network - CPN).

Service Search: The customer then searches for services in the market which may fulfil the requirements of the identified management tasks.

Negotiation: Having identified candidate management tasks to be delegated and a provider able to carry out the task, a negotiation process takes place between the customer and provider. During the negotiation the provider may gather information about the resources on the customer side, and the terms of the contract are settled. The negotiation process results in a contract documenting the commitments done between the customer and the provider. For the customer, contracts assist the representation and control of objectives and authority of external entities in its environment. For the provider, the contract is the base information for instantiation and installation of the appropriate components for service provisioning.

Choice of Service Provider: A Customer may find various Providers appropriate for the provision of the management tasks it wants to delegate. In this case the choice of Provider can be made after negotiating with each one and analysing the best contracts offered. This choice is taken by a human responsible at customer side as it may involve various technical and non-technical aspects which make it difficult to automatize. Decision support may be offered by the system.

\section{In-service}

Contract Administration: In the in-service phase the main activity is to enforce the agreements of the Contract. On the one side the provider will carry out the management activity delegated. On the other side, customers map contract information to underlying supporting structures (e.g. security, accounting) in order to guarantee that the provider uses no more than the rights conceded by the Customer. In case of contract violation the parties may have to dispute about the violation using the notary and adjudicator services. In case of violation situations, a penalty may be applied, and/or the service may be suspended. Contract administration may also encompass contract modification. The in-service phase finishes when contract cancellation is requested by one of the parties.

Post-service

Contract Abrogation: Post-service activities are related to the abrogation of the Contract, i.e. the agreements are no longer valid, and the Service Provider has no rights within the Customer Premises.

\subsection{Information Exchanged}

The information manipulated and exchanged among the involved parties comprehends:

- service representation in the service market; 
- the information for representing the information system of the customer (such that the provider can dynamically obtain this information during the negotiation);

- contract clauses, holding aspects of the contract under negotiation. As a focus of this paper, contracts are described in more detail.

\section{Contracts}

A contract is resulted from a negotiation process (among negotiator components mentioned bellow) and contains the information necessary to represent and control the co-operation among customer and provider. This encompasses:

- identification of the administrative domains involved and the roles they play;

- identification (information for authentication) to be used by a role when it uses resources from another role;

- the management service which is to be carried out by the provider, and which group of resources is to be managed;

- authorisations are necessary and restrictions imposed to the parties;

- form of delivery of the service provided (e.g. via an interface to a management component at customer premises);

- price of the service and payment form.

In order to hold this information, a contract is modelled as having a set of clauses, where clauses can be:

- definitive - serve to define terms used by other clauses of the contract, or to denote some information important to the contract (e.g. define groups of objects mentioned in the contract);

- motivative - serve to identify the intents of the roles involved in the contract;

- authoritative - serve to identify the authorisations of the roles in relation to groups of resources;

- prohibitive - serve to identify the prohibitions to the roles in relation to groups of resources;

- coordinative - related to the time when the service is provided;

- compensative - state the accounting and payment procedures adopted;

- punitive - present possible actions when other clauses are violated;

- abrogative - state the conditions or actions to be done in order to cancel the contract.

Given below is an example of a contract.

Definitive Clauses

1 Role Customer Customer-AdmDomain-Identificator

2 Role Provider Provider8-AdmDomain-Identificator

3 Service S8 ServiceType: NetworkConfigurationManagement

ServiceDescr.: This service enables to open and close end-to-end connections in a network...

Properties: TargetMIB : M4AF-NetworkElement

TargetEnvironment: [CORBA2 (OrbixWeb) ]

ServiceInterface : [ M4AF-Network ] 
4 Subject Provider subj1

5 Object- Target- NetworkElements + atmFabrics + atmCrossConnections Group Objects + vpCTPBidirectionals

$\underline{\text { Motivative Clause }}$

6 Subject subj1 Authoritative Clause

7 Subject subj1 Prohibitive Clause

8 Not Subject subj1 Operations delete, destroy ObjectGroup TargetObjects

Clauses 1 and 2 state which administrative domains play which roles in the relationship ruled by the contract. The roles 'Customer' and 'Provider' have known semantics, and are associated to identificators of the corresponding administrative domains. Clause 3 defines a service and its properties. As a definitive clause it defines that the use of the word 'S8' is related to the service definition (more than one service could be defined in the context of a contract). Clause 4 defines a 'subj'ect name to be associated with the provider role. This name will be used by the provider in order to access the customer premises. Clause 5 defines a group of objects named 'TargetObjects' to be composed by objects of the kinds informed in the third parameter. Clause 6 informs that the subject with name 'subj1' will provide the service 'S8' on the objects 'TargetObjects'. Clauses 7 informs what operations are authorized for 'subj1' to perform in 'TargetObjects'. And clause 7 inform that subjects which are not 'subj1' are prohibited from calling 'delete' or 'destroy' operations on 'TargetObjects'.

\subsection{Interacting Components}

The components communicating among the involved parties and their interaction are also modelled. This comprehends:

- information repositories carrying information about the customer premises;

- security and authority decision support components on the customer side;

- mechanisms for detecting and solving conflicts among managers;

- customer and provider negotiator components - further detailed bellow;

- and components for contract management (activation, deactivation, cancellation) are also identified.

These components are modelled considering interaction with market mechanisms (for instance: Trader, Directory, World-Wide-Web, Notary, etc.).

\section{Negotiator Components}

The negotiation takes place between two negotiator components representing the involved administrative domains (i.e.: customer and provider), and settles the terms under which their co-operation will take place. The result of a negotiation process will be a contract, or no agreement between the parties.

Provider and Customer negotiators may respectively offer services and invite for negotiation. During the negotiation, the negotiators cyclically dialogue in order to decide what clauses (presented above) are added to the contract. For this, the 
negotiator components support a set of methods for manipulating contracts and contract clauses, as depicted in the IDL definition bellow.

interface ContractNegotiator \{

ContractId beginOfContract(in ContractId ctId) raises(NotApplicable); void abortContract()

void closeContract()

void request(in Clause cl)

void counterRequest(in Clause cl)

void accept(in Clause cl)

void reject(in Clause cl, in RejectData Reason)

void suspend(in Clause cl) raises(NotApplicable); raises(NotApplicable); raises(NotApplicable); raises(NotApplicable); raises(NotApplicable); raises(NotApplicable); boolean tokenRequest(); raises(NotApplicable);

;

interface CustomerContractNegotiator : ContractNegotiator \{

void offerService(in AdministrativeDomainId ad, in ServiceInfo si, in ProviderContractNegotiator en ) raises(NotApplicable);

\}

interface ProviderContractNegotiator : ContractNegotiator \{ void negotiateInvitation(in AdministrativeDomainId ad, in ServiceInfo si, in CustomerContractNegotiator cn ) raises(NotApplicable);

\}

The manipulation of contracts is done through: 'beginOfContract', 'abortContract', 'closeContract'. These methods are used to respectively starting, aborting, and trying successful end of negotiation. After a 'beginOfContract', the contract clauses to be added to the contact are negotiated through: 'request' - requests a clause to be added to the contract; 'counterRequest' - used if the entity receiving a request wants to change the clause before accepting it in the contract (a 'counterRequest' can be responded with another 'counterRequest', it is a matter of the negotiators strategy to control loops on 'counterRequests'); 'accept' - stops the dialogue about a clause by accepting it; 'reject' - stops the dialogue about a clause by rejecting it (reject reasons may be inconsistencies in clause expressions, clause conflicts, excessive authority required, or refusal of any other feature, e.g. price, etc.); 'suspend' - stops the dialogue about a clause, and postpones the answer to be done at a later point in time (suspended clauses of a contract can be submitted to a human responsible for decision). The sequence of possible exchanges among two negotiation partners 'P1' and 'P2' is given in Figure 2. 


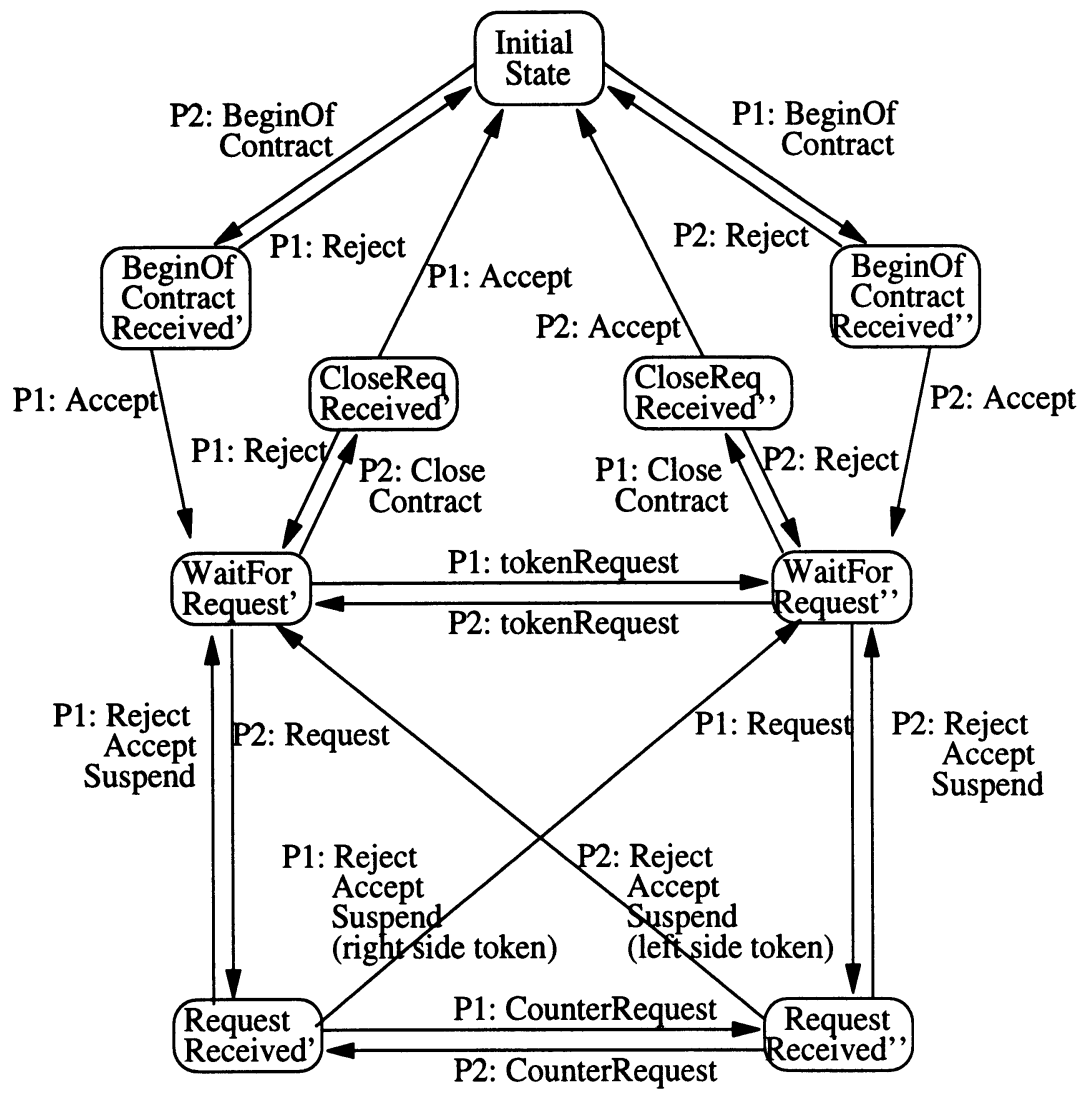

Obs.: any partner (P1 or P2), in any state after "BeginOfContract", may issue a request for "AbortContract". leading to the initial state.

Figure 2: State Machine for the Negotiation

\section{$4 \quad$ SUPPORT INFRASTRUCTURE}

In this section the requirements to the support infrastructure are briefly explained. Attention is focused on Customer side mechanisms because it is the domain where the managed resources are located and where the management service takes place. Furthermore, these requirements are important to measure the feasibility of the proposal done, because in the Customer side operational activities are to be minimised (alignment with management delegation goals).

\section{Environment Information}

In order for Providers to provide management services to previously unknown Customers, Customers must provide with information about the own resources. In the context of (ITU-T, 1993), the management information knowledge necessary for managers to recognize the capabilities of managed systems is proposed. In CORBA (OMG, 1993) conform environments, the Interface Repository and the 
Implementation Repository offer support to analogous information. Further support is needed to offer also relationship information, the contribution of the Meta Object Facility has to be evaluated for this purpose. In the scope of this work, the provider assumes known semantics to a known syntactic definition encountered in the environment information.

\section{Mobility}

If the communication between customer and provider is more intensive, then depending on the characteristics of the network providers used for the customerprovider communication, remote execution or migration of components can be used. In the negotiation phase, a provider negotiator component could be sent to the customer premises to acquire information about it, negotiate the service requested, and return to the provider with the contract - in this case migration could be used.

Migration or remote execution of components will lead to additional support for security, accounting. Security mechanisms are required to guarantee the secure execution of provider components at customer premises. Accounting mechanisms are likewise necessary in order to account the resources used by provider components at customer premises.

Mobile agents (Cristaliz et al., 1997) reveal to be useful to implement providers components which execute at customer premises, as the infrastructures for mobile agents address issues close to the ones here commented.

Security

Each entity performing in the customer premises will have a degree of authorization to use the services on the customer side. In order to guarantee that the actual service utilisation is allowed for the entity, authentication and access control mechanisms are necessary.

\section{Domains and Policies}

Domains and Policy mechanisms are useful to group resources in management domains and associate policies to them. These policies may represent authorizations and prohibitions of entities on the resources (thus supporting access control mechanisms) as well as regions of responsibility of providers over resources.

\section{IMPLEMENTATION}

The main components of the model were implemented in Java, for CORBA. A scenario for Network Management was chosen. At Customer side CORBA objects simulating network elements (ATM Switches) were implemented. Two management service providers were implemented which provide configuration and performance management services over the customer resources.

A subset of Domain and Policy structures following CORBA Security (OMG, 1995) were implemented due to the non availability of such structures at CORBA conform products during the prototype. Extensions for supporting other kinds of 
policies as well as domain membership administration were done. The access control function (which consults Domain and Policy structures for deciding access) was implemented.

In order to support contract management, a Contract Service was implemented and is responsible for creating and storing contracts. The Contract Service is used by the Contract Negotiators. A Customer Contract Negotiator and two Provider Contract Negotiators were implemented. The functionality of a Contract Negotiator was discussed above.

A Contract Administrator is responsible for activating the contract, after it is successfully negotiated. Contract activation results, on the customer side, in the mapping of contract clauses to supporting structures which guarantee that service operation will take place as agreed. For instance, authorisations and prohibitions are mapped to authentication and access control mechanisms. On the provider side, contract activation will lead to the creation and installation of components which implement the service. Mobile agents reveal to be useful to implement providers components which execute at customer premises.

The implementation has shown the feasibility of the model, offering high-level support and reducing the complexity for the customer. However, it shows also that further complementary mechanisms need to be developed in order to satisfactorily support the customer-provider co-operation. For instance: group communication (to address multi-party negotiation), passing objects by value (OMG, 1996c) among distributed objects; as well as a common framework for the definition of clauses and their semantics. Additional support in the service market like feedback mechanisms for evaluation of service providers is also necessary.

\section{CONCLUSIONS AND FUTURE ACTIVITIES}

The proposed mechanisms address in an uniform way the delegation of different types of management activities over different types of resources, so that the customer deals in a higher level of abstraction instead of handling in a specific way for each different case. Furthermore, these mechanisms, complemented by auxiliary roles from the electronic market (e.g.: trader), allow the customer to freely handle with various providers, and easily substitute contracted ones if necessary. With this the customer is able to delegate management tasks over its resources while maintaining autonomy in decisions related to the own premises.

The developed components have a close relationship with current activities in the field of electronic commerce (McConnell, 1997) (Merz, 1997) (OMG,1997). On the one hand, functions from the electronic commerce (e.g.: electronic payment (OMG, 1996a), notary) are needed to support the delegation of management activities in the open service market. On the other hand, some basic mechanisms proposed here can be generalised to be used in the context of electronic commerce. Future activities include: the generalisation of the developed mechanisms to address other types of co-operations; negotiation involving various parties; further investigation of distinct strategies which negotiators may use; as well as 
investigation of forms of enforcing different contract clauses using current computing platforms.

\section{Acknowledgements}

The author is grateful to GMD-Fokus (German Research Centre for Information Technology - Research Institute for Open Communicating Systems) for hosting his activities relative to this work.

\section{REFERENCES}

OMG (1993). Common Object Request Broker: Architecture and Specification. OMG Doc. No. 93.xx.yy. Rev.1.2. Draft Dec 29, 1993.

OMG (1995). CORBA Security. OMG Document 95-12-1. December 1995.

McConnell, Stephen (1997). The OMG/CommerceNet Joint Electronic Commerce Whitepaper. July 27, 1997. EC/97-06-09.

Merz, Michael et.al. (1997) OSM D9 Research Platform. February 17, 1997.

OMG (1997). Negotiation Facility RFP. OMG Document: ec/97-06-05.

OMG (1996a). Payment Facility RFP 1. OMG Document: ec/96-11-02.

OMG (1996b). Common Facilities RFP-4. Common Business Objects and Business Object Facility. OMG Doc. cf/96-01-04.

OMG (1996c). Objects by Value RFP. OMG Document: orbos/96-06-04.

Cristaliz; General Magic; GMD Fokus; IBM (1997). Mobile Agent Facility Specif. Joint Submission. June 2, 1997. cf/97-06-04.

ITU-T (1995). ITU-T Draft Rec. X.750 I ISO/IEC DIS 10164-16. Information Technology Open Systems Interconnection - Systems Management: Management knowledge management function. April 17, 1995.

\section{BIOGRAPHY}

Fernando Luís Dotti obtained his BSc. in 1989 and MSc. in 1992 from the University 'Universidade Federal do Rio Grande do Sul' - Brazil. In 1997 he obtained the PhD degree at the Technical University Berlin - Germany. Since march/1998 Fernando is a lecturer at the University 'Pontifícia Universidade Católica do Rio Grande do Sul' - Brazil. 